INTERNATIONAL JOURNAL OF RESEARCHES IN BIOSCIENCES, AGRICULTURE AND TECHNOLOGY (C) VISHWASHANTI MULTIPURPOSE SOCIETY (Global Peace Multipurpose Society) R. No. MH-659/13(N) www.vmsindia.org

\title{
LIST OF FRESHWATER MITOSPORIC FUNGI OF MADHYA PRADESH
}

\author{
Suhas A. Chaudhari' ${ }^{1}$ V. R. Patil ${ }^{2}$ and B. D. Borse ${ }^{3}$ \\ ${ }^{1}$ G.D. Benadale Mahila Mahavidyalya, Jalgaon \\ 2S.V.S.Naik A.C.S.College Raver \\ ${ }^{3}$ N.S.S., Dhule's Uttamrao Patil Arts \& Science College, Dahivel, Tal.- Sakri, Dist.- Dhule
}

\begin{abstract}
:
The present paper deals with distribution and substratum range of 362 species of freshwater Mitosporic fungi (346 Hyphomycetes and 16 Coelomycetes) reported so far from freshwater habitats of India. They were found as saprophytes on woody debris and leaf litter submerged in freshwater environment. Conidia of most of these fungi were encountered in foam samples. A checklist of freshwater Mitosporic fungi recorded from India is compiled on the basis of present studies in Maharashtra, Gujarat and Madhya Pradesh and published literature. Distribution of 362 species of freshwater Mitosporic fungi reported so far from various states of India is provided. The most frequently collected species of the genera are Camylospora, Canalisporium, Flabellospora, Lemonniera, Tetracladium, and Tricladium. The checklist includes detail of the location and substrata on which they encountered. This data will be useful in the compilation of freshwater fungal biodiversity of India.
\end{abstract}

Keywords: Freshwater, Mitosporic fungi, Submerged leaves, Foam samples.

\section{Introduction}

Freshwater fungi are defined as "fungi that for the whole or part of their life cycle rely on freshwater" (Thomos, 1996). Estimates for the number of fungi in the world range up to ca. 13.5 M species (Kirk et al., 2008). So far only 1.7 million species of organisms are known to the Science as against the estimated species on our planet. It indicates the large number of organisms are yet unknown to the Science (Hawksworth, 2001). Among the known 1.7 million species, 0.2 million $(13 \%)$ are reported from our country (Manoharachary et al., 2005).

In India, though these fungi are being studied at a very few states (Assam, Tamil Nadu, Andhra Pradesh, Goa, Karnataka, Kerala, Madhya Pradesh, Maharashtra, Gujarat), yet about 360 species have also been recorded (Natarajan and Udaiyan, 1978; Udaiyan and Hosogaudar, 1991; Udaiyan and Manian, 1991a, b; Tiwari, 1992; Sati and Tiwari, 1997; Sridhar et al., 1992; Manoharachary, 1989; Bhat et al., 2009; Sati and Joshi, 2011) including some new species (Sridhar and Kaveriappa, 1987; Rajashekhar et al., 1991; Udaiyan, 1991; Sati and Tiwari, 1992, 1993, 2003; Sati et al., 2009; Sridhar and Kaveriappa, 2002; Soosamma et al., 2001; Nair and Bhat, 2001, 2002a, b).

We have been investigating diversity of freshwater higher fungi that decay leaves and wood submerged in the rivers, streams, and lakes in the central part of India. Previous work on these fungi from Madhya Pradesh ware made by Hasija and Shanware (1986), Agrawal et al. (1989, 1991, 1992), Hasija and Singhal (1991) and Upadhyaya et al. (2012).
Previous work on these fungi from Maharashtra were made by Patil and Rao (1972), Patil and Kapadnis (1979), Thakur (1977), Patil (1998a, b, 2000, 2003a, b, 2007), Talde (1981, 1983), Shinde and Pawar (2008, 2009), Borse and Patil (2006, 2007); Borse et al. (2008, 2014); Patil (2009); Pawara et al. (2009, 2011); Wagh et al. (2009); Patil et al. (2011, 2012a,b); Patil and Borse (2011, 2012); Jadhav et al. (2011); Nemade et al. (2009, 2010); Nemade and Patil (2010); Ghanwat and Reddy (2011); and Wagh and Borse (2014). Previous work on these fungi from Gujarat was made by Ahire et al. (2009).

\section{Materials and Methods}

The samples of submerged woody debris, leaves and foam were collected from both the lentic and lotic habitats randomly during 2011-15 from different lentic and lotic habitats from Maharashtra, Gujarat and Madhya Pradesh. The following three methods were used for isolation of various fungal species.

Wood analysis: The samples woody debris were placed in plastic bags and sealed well in order to avoid moisture loss. On returning to the laboratory, samples with debris and fouling organisms were washed thoroughly with running tap water. Surface fouling organisms were scrapped off, following rinsing in tap water. The fresh samples were examined using a stereomicroscope for fungal growth. After initial observations, samples were incubated in plastic boxes and kept moist by spraying with distilled water and periodically examined for presence of fungal growth.

Leaf litter analysis: Submerge leaves of different kinds were collected randomly from 
sampling sites and brought to the laboratory in moist polythene bags. They were washed several times in tap water and finally in distilled water. They were cut into small bits and incubated separated in Petri dishes containing distill water at laboratory temperature $\left(25-30^{\circ} \mathrm{c}\right)$. The water was replaced in Petri dishes once in two days to minimize the growth of bacteria and other organisms. The leaf bits were screened under an inverted microscope at 24 hours intervals for 60 days to detect the water borne fungi appearing on them.

Foam analysis: In aquatic habitats, foam is formed by the movement of the water against natural barriers like stones, logs, twigs, especially in lotic habitats, constitutes a natural trap for the conidia of aquatic Hyphomycetes. Foam samples were collected at morning and evening time. Samples were placed in cleaned wide mouthed plastic bottles and kept for 24 hours to enable the foam to dissolve. It was prepared by adding FAA to yield 5\% foam solution. Then samples were brought to laboratory and scanned under low or high power of a microscope using $15 \mathrm{x}$ eyepiece for the presence of conidia of freshwater Mitosporic fungi.

Permanent voucher slides of fungi were prepared according to the 'double cover glass method' described by Volkmann-Kohlmeyer and Kohlmeyer (1996). Reports of fungi studied were confirmed with the help of Bilgrami et al. (1991),
Sridhar et al. (1992), Jamaluddin et al. (2004) and relevant literature.

\section{Results and Discussions:}

A list of 362 species of Freshwater Mitosporic fungi which have been identified to species level by various researchers of India. They were found as saprophytes on submerged leaves (179 sp.), Conifer needles (26 sp.), Root endophytes (19 sp.), submerged woody debris (204 sp.), water samples (64 sp.), conidia in stem flow (44 sp.) and conidia in foam samples (142 sp.) Most records of these fungi were from states of Madhya Pradesh (73 sp) in table represent intensity of studies on these fungi. As aquatic habitats are increasingly altered and degraded, it is imperative that the freshwater fungal species of the remaining high quality aquatic habitats be characterized and isolated. Such baseline information is essential to understand the role of fungi in aquatic habitats and how fungi could be used in the remediation of damaged aquatic habitats. It is clear those additional collections from worldwide, especially in tropical areas and along altitudinal gradients, are needed to fully characterize the biodiversity, geographical distribution pattern, systematics and evolution of freshwater Mitosporic fungi. In summary, we hope that the information presented herein will prompt future studies to document Freshwater Mitosporic fungi of India.

Table 1. Freshwater Mitosporic Fungi of India: (F-Foam, L- leaf, CN-Conifer Needles, RE-Root Endophytes, SFStem Flow, W-Water, WD-Wood; MP-Madhya Pradesh.

\begin{tabular}{|c|c|c|c|}
\hline $\mathbf{S r}$ & Name of species & Substrates & Location \\
\hline & Hyphomycetes & $\begin{array}{l}\mathrm{F}, \mathrm{L}, \mathrm{CN}, \mathrm{RE}, \\
\mathrm{SF}, \mathrm{W}, \mathrm{WD}\end{array}$ & $\begin{array}{l}\text { Madhya } \\
\text { Pradesh }\end{array}$ \\
\hline 1 & $\begin{array}{l}\text { Alatospora acuminata } \\
\text { Ingold }\end{array}$ & $\mathrm{F}, \mathrm{L}, \mathrm{CN}, \mathrm{W}$ & + \\
\hline 2 & $\begin{array}{l}\text { Alternaria } \\
\text { chlamydosporum } \\
\text { Mouch. }\end{array}$ & $\mathrm{L}, \mathrm{WD}$ & + \\
\hline 3 & $\begin{array}{l}\text { A. tenuissima (Hunze ex } \\
\text { Fr.) Wiltsh. }\end{array}$ & WD & + \\
\hline 4 & A. crassa Ingold & $\begin{array}{l}\mathrm{F}, \mathrm{L}, \mathrm{CN}, \\
\mathrm{RE}, \mathrm{W}, \mathrm{WD}\end{array}$ & + \\
\hline 5 & $\begin{array}{l}\text { A. longissima (Sacc. \& } \\
\text { P. Syd.) Ingold }\end{array}$ & $\begin{array}{l}\mathrm{F}, \mathrm{L}, \mathrm{RE}, \\
\mathrm{WD}\end{array}$ & + \\
\hline 6 & Aspergillus niger Tiegh. & $\mathrm{L}, \mathrm{WD}$ & + \\
\hline 7 & $\begin{array}{l}\text { Barnettela jabalpurensis } \\
\text { G.P. Agarwal et al. }\end{array}$ & $\mathrm{L}$ & + \\
\hline 8 & $\begin{array}{l}\text { Beltrania rhombica } \\
\text { Penz. }\end{array}$ & $\mathrm{F}, \mathrm{L}, \mathrm{W}$ & + \\
\hline 9 & $\begin{array}{l}\text { Camposporium } \\
\text { antennatum Harkness }\end{array}$ & $\mathrm{F}, \mathrm{L}$ & + \\
\hline 10 & Campylospora & F,CN,RE,W & + \\
\hline
\end{tabular}

\begin{tabular}{|c|c|c|c|}
\hline & chaetocladia Ranzoni & ,WD & \\
\hline 11 & $\begin{array}{l}\text { Camylospora filicladia } \\
\text { Nawawi }\end{array}$ & $\mathrm{F}, \mathrm{L}$ & + \\
\hline 12 & $\begin{array}{l}\text { Canalisporium caribense } \\
\text { (Hol.-Jech. \& Mercado.) } \\
\text { Nawawi \& Kuthub. }\end{array}$ & WD & + \\
\hline 13 & $\begin{array}{l}\text { Canalisporium exiguun } \\
\text { Goh \& Hyde }\end{array}$ & WD & + \\
\hline 14 & $\begin{array}{l}\text { Canalisporium pulchrum } \\
\text { (Hol.-Jech. \& Mercado) } \\
\text { Nawawi \& Kuthub. }\end{array}$ & WD & + \\
\hline 15 & $\begin{array}{l}\text { Ceratosporella deviata } \\
\text { Subram. }\end{array}$ & $\mathrm{L}$ & + \\
\hline 16 & $\begin{array}{l}\text { Cirrenalia indica Vasant } \\
\text { Rao \& Reddy }\end{array}$ & $\mathrm{L}$ & + \\
\hline 17 & $\begin{array}{l}\text { Cladosporium } \\
\text { cladosporioides (Fres.) } \\
\text { de Vries }\end{array}$ & WD & + \\
\hline 18 & $\begin{array}{l}\text { Cladosporium } \\
\text { cucumerinium Ellis \& } \\
\text { Arth. }\end{array}$ & $\mathrm{L}, \mathrm{WD}$ & + \\
\hline 19 & $\begin{array}{l}\text { Clavariopsis aquatica } \\
\text { De Wild. }\end{array}$ & $\begin{array}{l}\mathrm{F}, \mathrm{L}, \mathrm{CN}, \\
\mathrm{RE}, \mathrm{W}\end{array}$ & + \\
\hline 20 & Clavariopsis & $\mathrm{F}, \mathrm{L}, \mathrm{W}$ & + \\
\hline
\end{tabular}




\begin{tabular}{|c|c|c|c|}
\hline & Nawawi & & \\
\hline 21 & $\begin{array}{l}\text { Clavariopsis } \\
\text { brachycladia Tubaki }\end{array}$ & $\mathrm{F}, \mathrm{L}$ & + \\
\hline 22 & $\begin{array}{l}\text { Clavatospora } \\
\text { longibrachiata (Ingold) } \\
\text { Sv. Nils. ex Marvanova } \\
\text { \& Nils. }\end{array}$ & $\mathrm{F}$ & + \\
\hline 23 & $\begin{array}{l}\text { Curvularia lunata } \\
\text { (Wakker) Boedijin }\end{array}$ & $\mathrm{L}, \mathrm{WD}$ & + \\
\hline 24 & $\begin{array}{l}\text { Curvularia tuberculata } \\
\text { Jain }\end{array}$ & L,WD & + \\
\hline 25 & $\begin{array}{ll}\text { Dactylella } & \text { submersa } \\
\text { (Ingold) } & \text { S. Nilsson } \\
\end{array}$ & $\mathrm{F}, \mathrm{WD}$ & + \\
\hline 26 & $\begin{array}{l}\text { Dendrosporium lobatum } \\
\text { Plakidas \& Edgerton }\end{array}$ & $\mathrm{F}, \mathrm{L}$ & + \\
\hline 27 & $\begin{array}{l}\text { Dictyosporium elegans } \\
\text { Corda }\end{array}$ & $\mathrm{L}, \mathrm{WD}$ & + \\
\hline 28 & $\begin{array}{l}\text { Dimorphospora foliicola } \\
\text { Tubaki }\end{array}$ & $\mathrm{L}, \mathrm{CN}, \mathrm{WD}$ & + \\
\hline 29 & $\begin{array}{l}\text { Diplocladiella } \\
\text { appendiculata Nawawi }\end{array}$ & $\mathrm{F}, \mathrm{L}$ & + \\
\hline 30 & $\begin{array}{l}\text { Diplocladiella } \\
\text { longibrachiata Nawawi } \\
\text { \& Kuthub. }\end{array}$ & $\mathrm{F}, \mathrm{L}, \mathrm{W}$ & + \\
\hline 31 & $\begin{array}{l}\text { Diplocladiella } \\
\text { tricladioides Nawawi }\end{array}$ & $\mathrm{F}$ & + \\
\hline 32 & $\begin{array}{l}\text { Drechslera australiensis } \\
\text { (Bugnicourt) Subram. \& } \\
\text { Jain ex M.B. Ellis }\end{array}$ & $\mathrm{L}, \mathrm{WD}$ & + \\
\hline 33 & $\begin{array}{ll}\text { Drechslera } & \text { spicifer } \\
\text { Nelson } & \\
\end{array}$ & $\mathrm{L}, \mathrm{WD}$ & + \\
\hline 34 & $\begin{array}{l}\text { Flabelloladia tetracladia } \\
\text { (Nawawi) Nawawi }\end{array}$ & $\mathrm{F}, \mathrm{L}, \mathrm{W}$ & + \\
\hline 35 & $\begin{array}{l}\text { F. acuminata Descals \& } \\
\text { Webster }\end{array}$ & $\mathrm{F}, \mathrm{L}$ & + \\
\hline 36 & F. crassa Alasoad. & $\begin{array}{l}\text { F,L,SF, } \\
\text { WD }\end{array}$ & + \\
\hline 37 & F. multiradiata Nawawi & $\mathrm{F}, \mathrm{L}, \mathrm{SF}, \mathrm{W}$ & + \\
\hline 38 & $\begin{array}{ll}F . & \text { verticillata } \\
\text { Alasoadura } & \end{array}$ & $\begin{array}{l}\mathrm{F}, \mathrm{L}, \mathrm{CN}, \\
\mathrm{SF}\end{array}$ & + \\
\hline 39 & $\begin{array}{l}\text { Flagellospora curvula } \\
\text { Ingold }\end{array}$ & $\begin{array}{l}\text { F,L,SF, } \\
\text { WD }\end{array}$ & + \\
\hline 40 & F. penicillioides Ingold & $\begin{array}{l}\mathrm{F}, \mathrm{L}, \mathrm{CN}, \\
\mathrm{SF}, \mathrm{W}, \mathrm{WD}\end{array}$ & + \\
\hline 41 & F. prolifera Petersen & $\mathrm{F}$ & + \\
\hline 42 & $\begin{array}{l}\text { F. oxysporum Schl. ex } \\
\text { Fries }\end{array}$ & $\mathrm{L}, \mathrm{WD}$ & + \\
\hline 43 & $\begin{array}{ll}\text { Helicodendron } & \\
\text { triglitziensis } & \text { (Japp) } \\
\text { Linder } & \\
\end{array}$ & $\mathrm{L}$ & + \\
\hline 44 & $\begin{array}{l}\text { Helicoma conicodenatum } \\
\text { Linder }\end{array}$ & $\mathrm{F}, \mathrm{WD}$ & + \\
\hline 45 & $\begin{array}{l}\text { H. hyderabadensis Rao } \\
\text { \& Deo Rao }\end{array}$ & $\mathrm{L}$ & + \\
\hline 46 & H. roseus Link & $\begin{array}{l}\text { F,L,SF, } \\
\text { WD }\end{array}$ & + \\
\hline 47 & $\begin{array}{l}\text { Helicosporium griseum } \\
\text { Berk. \& Curtis }\end{array}$ & F,L,WD, & + \\
\hline 48 & $\begin{array}{l}\text { Hydrometrospora } \\
\text { symmetrica Gonczol \& } \\
\text { Revay }\end{array}$ & $\mathrm{F}$ & + \\
\hline
\end{tabular}

\begin{tabular}{|c|c|c|c|}
\hline 49 & $\begin{array}{l}\text { Isthmotricladia britanica } \\
\text { Descals }\end{array}$ & $\mathrm{L}$ & + \\
\hline 50 & $\begin{array}{l}\text { Isthmotricladia } \\
\text { gombakiensis Nawawi }\end{array}$ & $\mathrm{F}, \mathrm{L}, \mathrm{W}$ & + \\
\hline 51 & $\begin{array}{l}\text { Isthmotricladia laeensis } \\
\text { Matsushima }\end{array}$ & $\mathrm{F}, \mathrm{L}, \mathrm{SF}, \mathrm{W}$ & + \\
\hline 52 & $\begin{array}{l}\text { Jaculispora submersa } \\
\text { Hudson \& Ingold }\end{array}$ & $\mathrm{F}, \mathrm{L}$ & + \\
\hline 53 & $\begin{array}{l}\text { Laridospora } \\
\text { appendiculata } \\
\text { (Anastasiou) Nawawi }\end{array}$ & $\mathrm{F}, \mathrm{L}, \mathrm{W}$ & + \\
\hline 54 & $\begin{array}{l}\text { Lemonniera aquatica De } \\
\text { Wild. }\end{array}$ & $\mathrm{F}, \mathrm{L}, \mathrm{SF}, \mathrm{W}$ & + \\
\hline 55 & $\begin{array}{l}\text { Lunulospora curvula } \\
\text { Ingold }\end{array}$ & $\begin{array}{l}\mathrm{F}, \mathrm{L}, \mathrm{CN}, \mathrm{RE}, \\
\mathrm{SF}, \mathrm{W}, \mathrm{WD}\end{array}$ & + \\
\hline 56 & $\begin{array}{l}\text { Lunulospora } \\
\text { cymbiformis Miura }\end{array}$ & $\begin{array}{l}\text { F,L,CN,W, } \\
\text { WD }\end{array}$ & + \\
\hline 57 & $\begin{array}{l}\text { Monodictys levis } \\
\text { (Wiltshire) S. Hughes }\end{array}$ & WD & + \\
\hline 58 & $\begin{array}{l}\text { Neta angliae Hyde and } \\
\text { Goh }\end{array}$ & WD & + \\
\hline 59 & $\begin{array}{l}\text { Phalangispora constricta } \\
\text { Nawawi \& J. Webster }\end{array}$ & $\mathrm{F}, \mathrm{L}, \mathrm{SF}, \mathrm{W}$ & + \\
\hline 60 & $\begin{array}{l}\text { Phialogangaliospora } \\
\text { lignicola Udaiyan \& V.S. } \\
\text { Hosag. }\end{array}$ & WD & + \\
\hline 61 & $\begin{array}{l}\text { Sporoschisma saccadoi } \\
\text { E.W. Mason \& } 8 \text { S. } \\
\text { Hughes }\end{array}$ & WD & + \\
\hline 62 & $\begin{array}{l}\text { Sporoschisma } \\
\text { uniseptatum Bhat \& } \\
\text { W.B. Kendr. }\end{array}$ & WD & + \\
\hline 63 & $\begin{array}{l}\text { Stemphyliomma terricola } \\
\text { Manohar. \& P. Rama } \\
\text { Rao }\end{array}$ & WD & + \\
\hline 64 & $\begin{array}{l}\text { Tetracladium } \\
\text { marchalianum } \\
\text { Wildeman }\end{array}$ & $\begin{array}{l}\text { F,L,CN,RE, } \\
\text { W }\end{array}$ & + \\
\hline 65 & $\begin{array}{l}\text { Tetraploa aristata Berk. } \\
\& \mathrm{Br} .\end{array}$ & $\mathrm{F}, \mathrm{L}$ & + \\
\hline 66 & $\begin{array}{l}\text { Torula herbarum (Pers.) } \\
\text { Link ex Fries }\end{array}$ & $\mathrm{L}, \mathrm{WD}$ & + \\
\hline 67 & $\begin{array}{l}\text { Trichoderma viride Pers. } \\
\text { ex Fr. }\end{array}$ & L,WD & + \\
\hline 68 & $\begin{array}{l}\text { Tricladium splendens } \\
\text { Ingold }\end{array}$ & $\begin{array}{l}\text { F,L,CN,SF, } \\
\text { W }\end{array}$ & + \\
\hline 69 & $\begin{array}{l}\text { Triscelophorus } \\
\text { monosporus Ingold }\end{array}$ & $\begin{array}{l}\text { F,L,CN,SF, } \\
\text { W,WD }\end{array}$ & + \\
\hline 70 & $\begin{array}{l}\text { Tumularia aquatica } \\
\text { (Ingold) Descals \& } \\
\text { Marvanova }\end{array}$ & $\mathrm{F}, \mathrm{W}$ & + \\
\hline 71 & $\begin{array}{l}\text { Varicosporium } \\
\text { helicosporium Nawawi }\end{array}$ & $\mathrm{F}, \mathrm{L}$ & + \\
\hline 72 & $\begin{array}{l}\text { Varicosporium } \\
\text { scoparium Roldon \& } \\
\text { Honrubia }\end{array}$ & $\mathrm{F}$ & + \\
\hline 73 & $\begin{array}{l}\text { Wiesneriomyces } \\
\text { laurinus (Tassi) P. M. } \\
\text { Kirk }\end{array}$ & $\mathrm{F}, \mathrm{L}, \mathrm{W}, \mathrm{WD}$ & + \\
\hline
\end{tabular}




\section{Acknowledgments:}

We are thankful to the Principal G..D. Benadale Mahila Mahavidyalya, Jalgaon, Chairman, Navodaya Shaikshanic Sanstha, Dahivel, Maharashtra and Principal,S.V.S.Naik College Raver(M.S.) for providing laboratory facilities. Thank are due to Dr. Angel Aguirre-Sanchez and authorities of Smithsonian Tropical Research Institute, Washington, DC, USA for sending pdf files of rare research articles on aquatic fungi.

\section{References:}

Agarwal, G.P., Hasija, S.K. \& Mishra, R.P. (1989). Aquatic fungi from Jabalpur (Madhya Pradesh). In: Perspective in Aquatic Biology (ed. Khulbe, R.D.), Papyrus Pub. House, New Delhi, pp. 105112 .

Agarwal, G.P., Hasija, S.K., Agarwal, P. \& Pandey, A.K. (1991). Fungi associated with submerged decaying leaves and twigs from Jabalpur. Proc. Nat. Acad. Sci. India, 61: 121-125.

Agarwal, G.P., Hasija, S.K., Agarwal, P. \& Pandey, A.K., (1992). Seasonal occurrence of Aquatic Hyphomycetes in different aquatic habitats at Jabalpur. J. Ind. Bot. Soc., 71: 95-97.

Ahire, P.K., Borse, B.D. \& Patil, S.Y. (2009). Aquatic fungi from Dang District of Gujarat -I. In: Biodiversity, Sustainable Development and Human Welfare, (Eds. Nandan et al.), Pub. SSVPS's, Science College, Dhule, (M.S.), pp. 278283.

Bhat, D.J, Pratibha, J, Gawas, P, Sarita, K.Y. \& Swapnaja, D. (2009). Diversity of microfungi in the forest of Western Ghats in Goa and surrounding regions. In: "Plant and fungal biodiversity and bioprospeting" (eds. Krishnan, S. \& Bhat, D.J.), Broadway Book Centre Publishers and Distributors, Pangim, Goa, pp.117-133.

Bilgrami, K. S., Jamaluddin, S. and Rizwi, M. A. (1991) "Fungi of India". Today and Tomorrow's Printers and Publishers, New Delhi, pp. 798.

Borse, B.D. \& Patil, R.S. (2007). Aquatic fungi from North Maharashtra-I. Bioinfolet, 4: 101-104.

Borse, B.D. \& Patil, S.Y. (2006). Aquatic fungi from North Maharashtra-IV: BRI's, J. Adv. Sci. \& Tech. 9: 91-95.

Borse, B.D., Patil, V.R. \& Patil, S.Y. (2008). Aquatic fungi from Buldhana district (M.S.) - 1 . Bioinfolet, 5: 44-47.

Borse, B.D., Jagdale, P. E. \& S. Y. Patil, S. Y. (2014) Freshwater Higher Fungi from Pune district (M.S., India) - I: The Mitosporic genus Xylomyces. Bioinfolet, 11: (In Press).

Ghanwat, S.P. \& Reddy, P.G. (2011). Notes on Hyphomycetes from fresh water habitats in Ahmednagar district, Maharashtra (India). Bioinfolet, 8: 359-362.
Hasija, S.K. \& Shanware, V. (1986). Aquatic Hyphomycetes of India - I. Two new records. Proc. Nat. Acad. Science India, 56: 74-76

Hasija, S.K. \& Singhal, P.K. (1991). Degradation of plant litter by aquatic Hyphomycetes. In: Handbook of applied mycology: soils and plants (eds, Arora, D.K., Rai, B, Mukerji, K.S. and Knudsen, G.), Marcel Dekker, Mew York, pp. 481505.

Hawksworth, D. L. (2001) The magnitude of fungal diversity: the 1.5 million species estimate revised. Mycol. Res., 105: 1422-1432.

Jadhav, C.S., Patil, S.Y. \& Borse, B.D. (2011). Aqutic fungi from Nashik district -1 . Recent Res. Sci. Tech., 3: 17-19.

Kirk, P.M., Cannon, P.F., Minter, D.W. \& Stalpers, J.A. (2008) Ainsworth and Bisby's Dictionary of the Fungi, 10 th Ed., CABI Publishing.

Jamaluddin, S., Goswami, M. G. and Ojha, B. M. (2004) “Fungi of India" (1989-2001), Scientific Publishers (India) Jodhpur, pp. 308.

Manoharachary, C. (1989) Glimses on WaterBorne conidial fungi from India. In: Perspective in Aquatic Biology (ed. Khulbe, R. D.), Papyrus Pub. Hou., New Delhi, pp. 71-77.

Manoharachary, C., Sridhar, K. R., Singh, Reena, Adhooleya, Alok, Suryanayanan, T. S., Rawat Seema \& Johshi, B. M. (2005) Fungal Diversity: Distribution, conversation and Prospecting of fungi from India. Curr. Sci., 89: 58-71.

Nair, S.K. \& Bhat, D.J. (2001) Diplospora indica, a new species of Hyphomycetes. Mycotaxon, 80: 101-104.

Nair, S.K. \& Bhat, D.J. (2002a) Kumbhamaya jalapriya, a new endophytic Hyphomycetes from India. Mycotaxon, 84: 65-68.

Nair, S.K. \& Bhat, D.J. (2002b) Dendrospora yessemreddea sp. nov., from freshwater foam. In: Frontiers in Microbial Biotechnology and Plant Pathology (eds. Manoharachary, C., Purohit, D.K., Rama Reddy, S., Singaracharya, M.A. \& Girishan, S.), Scientific Publishers, Jodhapur, pp. 295-298.

Natarajan, K. \& Udaiyan, K. (1978) Cooling tower fungi in India. Int. Biodeterior. Bull., 14: 85-87.

Nemade, L.C. \& Patil, V.R. (2010). Aquatic Fungi from Melghat-II. Res. Link, 78: 12-14.

Nemade, L.C., Patil, V.R. \& Borse, B.D. (2009). Aquatic fungi from Melghat - I. In: Biodiversity, Sustainable Development \& Human Welfare (eds. Nandan S.N. et al.,), SSVPS's Sci. College, Dhule (M.S.), pp. 191-195.

Nemade, L.C., Patil, V.R., Patil, M.S. \& Chaudhary, S.A. (2010). Diversity of freshwater fungi hyphomycetes from Buldhana district (M.S.), India. J. Ecobiotech., 2: 17-20.

Patil, A.S. \& Rao, V.G. (1972). Articulospora- a new record from India, Indian Phytopathology, 25: $455-456$. 
Patil, N.N. (1998a). Aquatic hyphomycetes of Mahabaleshwar. Geobios New Reports, 17: 90.

Patil, N.N. (1998b). Taxo-Ecological studies on some aquatic Hyphomycetes from India. In: Frontiers in Botany, Proc. Conf. on Modern trends in teaching and research in Botany, Pub. V.N. Mahavidyalaya, Aurangabad, M.S., India. pp. 8489.

Patil, N.N. (2000) Occurrence of aquatic conidial fungi on Memecylon umbelatum Burm. In: Plant Resource Development, Pub. Dept. of Botany, Dr. B.A.M. University, Aurangabad, M.S., India. pp. 83-85.

Patil, N.N. (2003a). Studies on aquatic Hyphomycetes associated with submerged leaves and foam in Maharashtra. Geobios, 30: 105-108.

Patil, N.N. (2003b). Aquatic Hyphomycetes from Western Ghats. In: Recent Adavances in Plant Microbial and Environmental Biotechnology (ed. N.N. Patil), Proc. of National conf., Pub. C.T. Bora College, Shirur, M.S., India. pp. 225-232.

Patil, N.N. (2007) A new host record of Ingoldiella hamata Shaw on Memecylon umbellatum Burm. J. Mycol. Pl. Pathol., 37: 345.

Patil, S.D. \& Kapadnis, B.P. (1979). Stream spora of Maharashtra. M. V. M. Patrika, 14: 59-64.

Patil, S.Y. (2009). Aquatic fungi from North Maharashtra-III. In: Biodiversity, Sustainable Development and Human Welfare, (Eds. Nandan et al.), Pub. SSVPS's, Sci. College, Dhule, (M.S.), pp. 76-81.

Patil, S.Y. (2012). Freshwater Ascomycetes from North Maharashtra - III. International Multidisciplinary Res. J., 2: 18-21.

Patil, S.Y. \& Borse, B.D. (2011). Aquatic fungi from North Maharashtra-VII. Recent Res. Sci. and Tech., 3: 8-11.

Patil, S.Y. \& Borse, B.D. (2012). Dematiaceous Hyphomycetes from North Maharashtra. International Multidisciplin. Res. J., 2: 36-38.

Patil, S.Y., Wagh, D.D. \& Borse B.D. (2012). Hyphomycetes from north Maharashtra. Current Botany, 3: 23-25.

Patil, V.R., Patil, S.Y., Nemade, L.C. \& Borse, B.D. (2011). Aquatic fungi from Buldhana district. Curr. Bot., 2: 56-58.

Patil V R, Patil S Y \& Borse B D (2011) Aquatic fungi from Buldhana District (M. S). Current Botany, 2: 56-58.

Patil, VR, Nemade, LC, Patil, SY \& Borse, B. D. (2012a) Studies on freshwater Hyphomycetes from Mangrul Dam, Dist.-Jalgaon (MS, India). In: The Proceedings of the "National Conference on Biodiversity, Biotechnology and Climate Change" (eds. Tuwar, AR \& Shaikh, MJ), 26-28 July, 2012, organized by ACS College, Sonai, Dt. Ahmednagar, Maharashtra, pp. 59-62.
Patil, S. Y., Wagh, D, D. \& Borse, B. D. (2012b) Hyphomycetes from North Maharashtra. Current Botany, 3: 23-25.

Pawara, C.M., Patil, S.Y. \& Borse B.D. (2011). Aquatic fungi from North Maharashtra-III. Bioinfolet, 8: 18-21.

Pawara, C.M., Patil, S.Y., Ahire, P.K. \& Borse, B.D. (2009). Aquatic fungi from North Maharashtra-V. In: Biodiversity, Sustainable Development and Human Welfare, (Eds. Nandan et al.), Pub. SSVPS's, Sci. College, Dhule, (M.S.), pp. 411-415.

Rajshekar, M., Bhat, D.J. \& Kaveriappa, K.M. (1991). An undescribed species of Vermispora from India. Mycologia, 83: 230-232.

Sati, S.C. \& Joshi, S. (2011). Aquatic Hyphomycetes and its anamorph (asexual stage) - Telomorph (sexual stage) relations: A review. The International J. Plant Reproduction Biol., 3: 89-98.

Sati, S.C. \& Tiwari, N. (1992). A new species of Tricladium from Kumaun Himalaya, India. Mycol. Res., 96: 229-232.

Sati, S.C. \& Tiwari, N. (1993). A new species of Pestalotiopsis on submerged leaf litter. Nova Hedwigia, 56: 543-547.

Sati, S.C. \& Tiwari, N. (1997). Glimpses of conidial aquatic fungi in Kumaun Himalaya. In: Himalayan Microbial Diversity, Vol.-I (eds. Sati, S.C., Saxena, J. \& Dubey, R.C.), Today and Tomorrow's Prints. and Publishers, New Delhi, pp. 17-33.

Sati, S.C. \& Tiwari, N. (2003). A new of Pleurophragmium from Nainital, Kumaun Himalaya, India. Science letters, 7 \& 8: 208-209.

Sati, S.C., Arya, P. \& Belwal, M. (2009). Tetracladium nainitalensis $\mathrm{sp}$. nov. a root endophyte from Kumaun Himalaya, India. Mycologia, 101: 692-695.

Shinde, V. \& Pawar, A.B. (2008). Some aquatic Hyphomycetes from Satara District. Bioinfolet, 5: 423-424.

Shinde, V. \& Pawar, A.B. (2009). Some aquatic Hyphomycetes from Satara District (M.S.). Geobios, 36: 161-164.

Soosamma, M., Lekha, G., Sreekala, K.N. \& Bhat, D.J. (2001). A new species of Trinacrium from submerged leaves from India. Mycologia, 93: 1200-1202.

Sridhar, K.R. \& Kaveriappa, K.M. (1987). A new species of Triscelosphorus. Indian Phtopath., 30: 102-105.

Sridhar, K.R. \& Kaveriappa, K.M. (2002). Synnematophora, A new aquatic Hyphomycete from the Western Ghat forests, India. Indian Jour. of Forestry, 25: 89-93.

Sridhar, K.R., Chandrashekar, K.R. \& Kaveriappa, K.M. (1992). Research on the Indian 
subcontinents. In: The Ecology of aquatic Hyphomycetes (Eds. Barlocher), Spinger-Varlag., Heidelbery Press, New York, pp. 182-211.

Talde, U.K. (1981). Aquatic deuteromycetous fungi from Purna Dudhna rivers. Ind. J, Mycol. \& Pl. Pathol., 11: 288-290.

Talde, U.K. (1983). Aquatic Hyphomycetes from Aundha Nagnath. Ind. Jour. Mycol. \& Pl. Patho., 13: $198-199$.

Thakur, S.B. (1977). Survival of some aquatic Hyphomycetes under dry condition. Mycologia, 69: $843-845$.

Tiwari, N. (1992). Taxonomy and species composition of Hyphomycetes in forest streams and their colonization pattern on tree leaves in Naini Tal, Central Himalaya. Ph. D. Thesis, Kumaun University, Naini Tal.

Thomas, K. (1996) Fresh water fungi. In: Introductory Volume to the Fungi. Part $2 \mathrm{C}$, Fungi of Australia, Vol. 1B, ABRS (ed. Grgurinovic), pp. $1-37$.

Udaiyan, K. (1991). Some interesting Hyphomycetes from industrial cooling Towers of Madras-I: J. Econ. Tax. Bot., 15: 627-647.

Udaiyan, K. \& Hosagoudar, V.S. (1991). Some fungi from the industrial water cooling Toweres of Madras-II: J. Econ. Tax. Bot., 15: 649-665.
Udaiyan, K. \& Manian, S. (1991a). Fungi deteriogens from preservative treated service timber packing in water cooling towers. Intern. Biodeteri. Bull., 27: 275-279.

Udaiyan, K. \& Manian, S. (1991b). Fungi colonizing wood in the Cooling tower water system at the Madras fertilizer company, Madras, India. Intern. Biodeteri. Bull., 27: 351-371.

Upadhyaya, A., Singh, J., Tiwari, J. \& Gupta, S. (2012). Biodiversity of water borne conidial fungi in Narmada River. International Multidisciplinary Res. J., 2: 20-22.

Volkmann-Kohlmeyer, B. \& Kohlmeyer, J. (1996). How to prepare truly permanent microscopic slides. Mycologist, 10: 107-108.

Wagh, S.N., Borse, B.D. \& Patil, S.Y. (2009). Aquatic fungi from north Maharashtra - VI. In: Biodiversity, Sustainable Development and Human Welfare, (Eds. Nandan et al.), Pub. SSVPS's, Sci. College, Dhule, (M.S.), Dhule, pp. 315-319.

Wagh, S. N. and Borse, B. D. (2014) Aquatic fungi from North Maharashtra- VIII. Indian Streams Res. J., 4: 1-4. 